\title{
Refractory Status Epilepticus Associated with Anti-SSA (anti-Ro) Antibodies
}

\author{
Jeremy J. Moeller, Daniel Friedman, Patricia Dugan, Cigdem I. Akman
}

Can J Neurol Sci. 2012; 39: 660-663

There have been frequent recent descriptions of limbic encephalitis and medically refractory epilepsy secondary to antibodies to a broad variety of neuronal antigens, including $\mathrm{N}$ methyl-D-aspartate receptors (NMDAR), glutamic acid decarboxylase (GAD) and voltage-gated potassium channels (VGKC). ${ }^{1}$ As a result, an intensive search for autoantibodies has become part of the diagnostic evaluation for unexplained refractory status epilepticus. We present two cases of sudden, severe limbic encephalitis and medically refractory status epilepticus associated with anti-SSA (Sjogren's-syndrome antigen A) antibodies, and discuss the potential implications of the diagnosis and management of new onset, cryptogenic status epilepticus.

\section{CASe Reports}

\section{Patient \#1}

A 27-year-old Hispanic woman with an unremarkable medical history presented with a two-day history of malaise, headache, fatigue and fever. On the day of admission she had frequent generalized tonic-clonic seizures. She was treated with lorazepam and phenytoin but continued to have seizures. She was intubated and placed on a continuous propofol infusion. A lumbar puncture demonstrated a mild lymphocytic pleocytosis and she was empirically started on acyclovir, ceftriaxone and vancomycin. Seizures continued, and carbamazepine was added to her regimen but a rash developed and it was discontinued. She was transferred to an academic medical center one week later for management of refractory status epilepticus. An electroencephalogram (EEG) performed on transfer showed nearly continuous generalized and bilaterally independent epileptiform discharges at 2-4 Hz, consistent with electrographic status epilepticus. Midazolam and pentobarbital continuous infusions were added and titrated to achieve EEG burst suppression.

A magnetic resonance image (MRI), performed on transfer, revealed bilateral mesial temporal fluid-attenuated inversion recovery (FLAIR) signal abnormalities with increased volume of both hippocampi (Figure). Lumbar puncture revealed 12 WBCs per $\mathrm{mm}^{3}$, normal protein and glucose, and positive oligoclonal bands. Her antinuclear antibody titer was elevated at $>1: 160$ (normal <1:40). SS-A (Ro) and SS-B (La) antibodies were highly elevated at 15616 and $1664 \mathrm{U} / \mathrm{mL}$ (normal <20 U/mL), respectively. Remaining evaluation for infectious, autoimmune and paraneoplastic abnormalities was negative (Table). Computed tomography of the chest, abdomen and pelvis and pelvic ultrasound revealed no abnormalities.

She was treated with a course of intravenous methylprenisolone $1 \mathrm{gm}$ /day for five days followed by plasma exchange for a total of five exchanges. She continued to require infusions of propofol, midazolam, and pentobarbital to control electrographic seizures despite the addition of levetiracetam, lacosamide, topiramate and phenobarbital. She underwent a trial of therapeutic hypothermia for 48 hours to control status epilepticus three weeks after presentation. She was weaned off all continuous infusions by five weeks after presentation. Shortly thereafter she was able to open her eyes in response to voice. Six weeks after presentation, she was able to follow commands and communicate with her family in Spanish. She had several isolated left temporal onset seizures in the setting of low phenytoin levels. She was transferred to a rehabilitation facility.

\section{Patient \#2}

A 10-year-old boy of Korean heritage with an unremarkable medical history presented with a three day history of fever, headache and abdominal discomfort. He was found unresponsive by his mother on the day of admission and was brought to the emergency department in a comatose state. He was empirically started on acyclovir, ceftriaxone and vancomycin. Electroencephalogram showed continuous focal seizures arising from both temporal lobes and so he was treated with intravenous lorazepam and levetiracetam, followed by intravenous pentobarbital. He was transferred to an academic medical center five days after the onset of symptoms. Neurological examination did not reveal any focal abnormalities. His initial EEG showed moderate diffuse slowing with frequent bilaterally independent sharp waves in both temporal regions and frequent intermittent electrographic seizures arising from the right temporal region. This progressed to a pattern of continuous generalized periodic discharges. Seizures persisted despite the treatment of phenobarbital, pentobarbital, levetiracetam and valproic acid.

Magnetic resonance image of the brain revealed increased size and increased T2/FLAIR signal in both hippocampi (Figure). Lumbar puncture was probably traumatic, with 16 white blood cells per $\mathrm{mm}^{3}$ and 10,700 red blood cells per $\mathrm{mm}^{3}$. Glucose was $87 \mathrm{mg} / \mathrm{dl}$ (serum glucose $114 \mathrm{mg} / \mathrm{dl}$ ) and protein of

From the Department of Medicine (Neurology), Dalhousie University (JJM), Halifax, NS, Canada; Department of Neurology (DF, PD), NYU Langone Medical Center; Department of Neurology (CIA), Columbia University Medical Center, New York, New York, USA.

Received January 9, 2012. Final Revisions Submitted March 6, 2012 Correspondence to: Jeremy Moeller, Suite 504, 44/46 Portland Street, Dartmouth, Nova Scotia, B2Y 1H4, Canada. Email: jmoeller@dal.ca. 

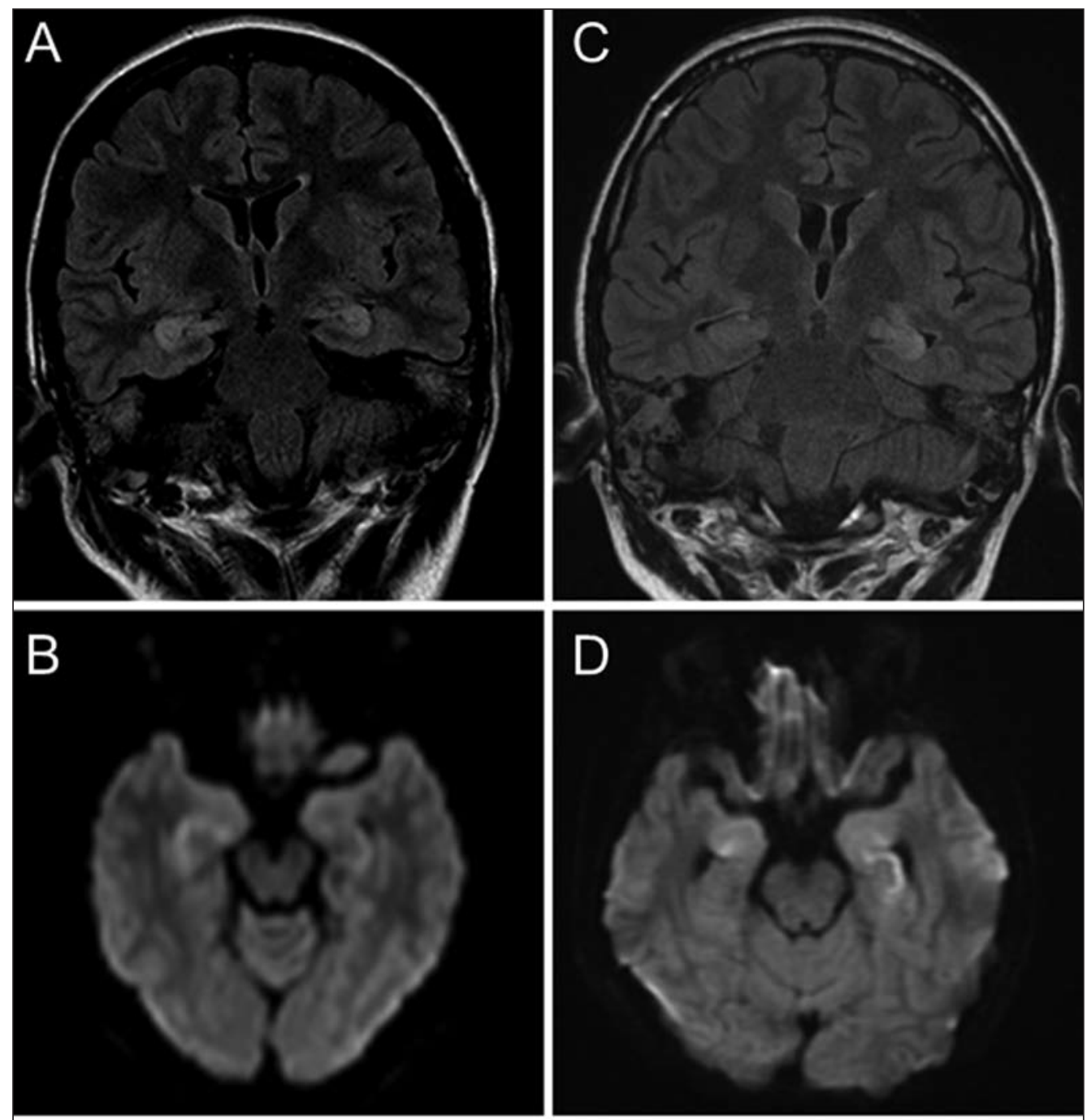

\section{Patient \#1}

\section{Patient \#2}

Figure: Brain magnetic resonance imaging of Patient \#1 one week after onset of seizures $(A, B)$ and Patient \#2 10 days after the onset of seizures $(C, D)$. The sequences include FLAIR $(A, C)$ and diffusion-weighted imaging $(B, D)$. In both patients, there was increased volume and increased FLAIR signal in the hippocampi bilaterally, and an area of restricted diffusion in the right anterior hippocampus.

$57 \mathrm{mg} / \mathrm{dl}$. Serum anti-nuclear antibodies and anti-DNA antibodies were negative, but qualitative anti-SSA antibodies were positive (quantitative test was repeated approximately one month after treatment, and was elevated at $90 \mathrm{AU} / \mathrm{mL}$; normal < $40 \mathrm{AU} / \mathrm{mL}$ ). Anti-glutamic acid decarboxylase antibodies were minimally elevated at $5.4 \mathrm{IU} / \mathrm{mL}$ (normal $<5.0 \mathrm{IU} / \mathrm{ml}$ ). The remainder of the laboratory evaluation for autoimmune, paraneoplastic, and infectious etiologies was negative (Table). Computed tomography of the chest, abdomen and pelvis did not reveal any definite abnormalities, and scrotal ultrasound was normal.

Ten days after presentation, he was given a single dose of intravenous gammaglobulin $(2 \mathrm{~g} / \mathrm{kg})$ followed by a five day course of intravenous methylprednisolone (1000 mg/day). Plasmapheresis was initiated approximately three weeks after presentation and the patient received a total of five treatments over the next ten days. After the first treatment with plasmapheresis, there was significant improvement in the EEG. Over the next one to two weeks, pentobarbital was slowly tapered, and the patient slowly began to regain consciousness. A month later, the patient developed a diffuse maculopapular rash and eosinophilia, and so phenobarbital was discontinued, resulting in several breakthrough seizures. By the time he was discharged to a rehabilitation facility, almost four months after presentation, he was alert and fully oriented, had normal language function, and could walk steadily without assistance. 
Table: Laboratory findings in two patients with limbic encephalitis and medically refractory epilepsy with anti-SSA antibodies

\begin{tabular}{|c|c|c|c|}
\hline & Patient 1 & Patient 2 & Reference Range \\
\hline \multicolumn{4}{|l|}{ Routine CSF analysis } \\
\hline White blood cell count & 12 per $\mathrm{mm}^{3}$ & 57 per $\mathrm{mm}^{3}$ (traumatic) & \\
\hline Red blood cell count & 55 per $\mathrm{mm}^{3}$ & 10,700 per $\mathrm{mm}^{3}$ (traumatic) & \\
\hline Protein & $30 \mathrm{mg} / \mathrm{dL}$ & $57 \mathrm{mg} / \mathrm{dL}$ & \\
\hline Glucose & $\begin{array}{l}88 \mathrm{mg} / \mathrm{dL} \text { (serum } \\
138 \text { ) }\end{array}$ & $87 \mathrm{mg} / \mathrm{dL}$ (serum 114) & \\
\hline
\end{tabular}

CSF Autoimmune/Inflammatory Indices

\begin{tabular}{|c|c|c|c|}
\hline IgG Index & 0.83 & Not tested & $0.28-0.66$ \\
\hline Oligoclonal bands & Positive & Not tested & \\
\hline Anti-NMDAR abs & Negative & Not tested & \\
\hline Anti-GAD abs & Negative & Not tested & \\
\hline \multicolumn{4}{|c|}{ Serum Autoimmune/Inflammatory Indices } \\
\hline Anti-NMDA abs & Negative & Negative & \\
\hline Anti-VGKC abs & Negative & Negative & \\
\hline Anti-neuronal nuclear abs & Negative & Negative & \\
\hline Anti-glial nuclear abs & Negative & Negative & \\
\hline Anti-Purkinje cell abs & Negative & Negative & \\
\hline Anti-CMRP-5 abs & Negative & Negative & \\
\hline Anti P/Q-type calcium channel abs & Negative & Negative & \\
\hline Anti-N-type calcium channel abs & Negative & Negative & \\
\hline Anti-GAD65 abs & Negative & $5.4 \mathrm{IU} / \mathrm{mL}$ & $<5.0 \mathrm{IU} / \mathrm{mL}$ \\
\hline Antinuclear antibody titer & $>1: 160$ & Negative & $<1: 40$ \\
\hline SS-A (anti-Ro) abs & $15616 \mathrm{U} / \mathrm{mL}$ & Positive (qualitative) & $<20 \mathrm{U} / \mathrm{mL}$ \\
\hline SS-B (anti-La) abs & $1664 \mathrm{U} / \mathrm{mL}$ & Negative & $<20 \mathrm{U} / \mathrm{mL}$ \\
\hline Anti-Sm abs & Negative & Negative & \\
\hline Anti-dsDNA abs & Negative & Negative & \\
\hline Anti-centromere abs & Negative & Not tested & \\
\hline anti U1RNP & Not tested & Negative & \\
\hline Anti-Scl-70 abs & Negative & Negative & \\
\hline Anti-Jo-1 abs & Not tested & Negative & \\
\hline Rheumatoid factor & Negative & Negative & \\
\hline Anti-thyroperoxidase & Negative & Negative & \\
\hline \multicolumn{4}{|c|}{ Serum Infectious Disease Evaluation } \\
\hline Influenza Virus A \& B PCR & Not tested & Negative & \\
\hline Parainfluenza virus PCR & Not tested & Negative & \\
\hline HIV PCR & Negative & Not tested & \\
\hline Serum Bartonella IgG & Not tested & Negative & \\
\hline \multicolumn{4}{|l|}{ CSF Infectious Disease Evaluation } \\
\hline Gram Stain \& culture & Negative & Negative & \\
\hline Fungal culture & Negative & Negative & \\
\hline Acid-fast bacilli stain & Negative & Negative & \\
\hline Herpes Simplex Virus PCR & Negative & Negative & \\
\hline Cytomegalovirus PCR & Negative & Negative & \\
\hline Varicella Zoster Virus PCR & Negative & Negative & \\
\hline Epstein Barr Virus PCR & Negative & Negative & \\
\hline
\end{tabular}

CSF = Cerebrospinal fluid; abs = antibodies; NMDAR = N-methyl-D-aspartate receptor; GAD = glutamic acid decarboxylase; VGKC = voltage-gated potassium channel; CRMP-5 = collapsing response mediated protein; dsDNA = double-stranded DNA; $\mathrm{PCR}=$ polymerase chain reaction test. 


\section{Discussion}

There are a number of similarities between these two cases, including a two to three day prodrome of fever, headache and malaise, followed by rapid onset of relentless clinical and electrographic status epilepticus refractory to treatment with multiple anti-epileptic drugs. Both patients also had at least one episode of systemic hypersensitivity reaction to anti-epileptic drugs. The patients demonstrated similar abnormalities on MRI, including increased size and increased FLAIR signal in both hippocampi. In both cases, several immune-modulating treatments were tried before the patients improved. Both patients had high titers of anti-SSA antibodies, and both had an extensive evaluation to rule out other, more common causes of status epilepticus. Patient \#2 had slightly elevated levels of antiglutamic acid decarboxylase antibodies, but limbic encephalitis and seizures are generally associated with much higher levels. ${ }^{2}$

Anti-SSA antibodies are antinuclear antibodies that are found most commonly in the sera of patients with Sjogren's syndrome and systemic lupus erythematosus, but they can be associated with other autoimmune diseases, including polymyositis/ dermatomyositis, rheumatoid arthritis and scleroderma. ${ }^{3}$ These antibodies can also be detected in approximately $3 \%$ of asymptomatic individuals, but it is possible that some of these individuals may go on to develop clinical features of autoimmune disease at some point in the future. ${ }^{4}$

The broad variety of central and peripheral nervous system manifestations of Sjogren's syndrome is well described. The exact prevalence of neurological manifestations in primary Sjogren's syndrome is disputed, but approximately $20-25 \%$ of patients with Sjogren's syndrome have some involvement of the nervous system. ${ }^{5}$ Seizures have been infrequently reported in association with Sjogren syndrome. Alexander et $\mathrm{al}^{6}$ first made the association between central nervous system dysfunction and Sjogren's syndrome in eight patients, of whom one had recurrent focal-onset seizures. They also reviewed prior reports of Sjogren's syndrome to date, and identified nine other reports of central nervous system involvement, including three reports of patients with seizures. Subsequent case reports of seizures associated with Sjogren's syndrome have been rare, and the underlying cause of the seizures has remained largely unexplained. In the largest series of patients with neurological manifestations of Sjogren's syndrome, 7/82 (8.5\%) had seizures. ${ }^{5}$ We are aware of only one published report of status epilepticus secondary to primary Sjogren's syndrome. ${ }^{7}$ This patient recovered from status epilepticus only after treatment with immune modulating therapy, and her seizures recurred when corticosteroids were tapered.

The clinical diagnosis of Sjogren's syndrome requires symptoms and objective evidence of lachrymal and salivary gland dysfunction and the presence of autoantibodies such as anti-SSA or anti-SSB.$^{5}$ Our patients have not yet been diagnosed with Sjogren's syndrome, and they have not yet demonstrated typical symptoms or signs. In one series, over half of patients with Sjogren's syndrome presented with neurological symptoms, and these symptoms often preceded sicca symptoms by a period of several years. ${ }^{5}$ Neurological symptoms were more likely to be the presenting feature in patients with central nervous system involvement compared to those with peripheral nervous system involvement. An alternative explanation is that the anti-SSA antibodies were simply an epiphenomenon of a more nonspecific immune reaction. The MRI findings of increased size and increased FLAIR signal in both hippocampi are non-specific and are similar to those seen in autoimmune limbic encephalitis associated with any of a large number of autoantibodies. ${ }^{1}$

Although there are no controlled trials of treatment of central nervous system manifestations of Sjogren's syndrome, case reports and case series suggest that some patients will improve or stabilize after treatment with corticosteroids. ${ }^{5,7}$ In our patients, aggressive treatment with corticosteroids and intravenous gammaglobulin was ineffective, and both patients required plasma exchange before achieving sustained improvement.

In conclusion, we believe that anti-SSA and anti-SSB antibodies should be considered the diagnostic evaluation of patients with unexplained medically-refractory status epilepticus. If identified, our experience suggests that aggressive immunomodulating therapy, including plasma exchange, may be useful in achieving seizure remission.

\section{REFERENCES}

1. Dalmau J. Status epilepticus due to paraneoplastic and nonparaneoplastic encephalitides. Epilepsia. 2009;50(Suppl 12): $58-60$.

2. Liimatainen S, Peltola M, Sabater L, et al. Clinical significance of glutamic acid decarboxylase antibodies in patients with epilepsy. Epilepsia. 2010;51:760-7.

3. Peene I, Meheus L, Veys EM, De Keyser F. Diagnostic associations in a large and consecutively identified population positive for anti-SSA and/or anti-SSB: the range of associated diseases differs according to the detailed serotype. Ann Rheum Dis. 2002; 61:1090-4.

4. Arbuckle MR, McClain MT, Rubertone MV, et al. Development of autoantibodies before the clinical onset of systemic lupus erythematosus. N Engl J Med. 2003;349:1526-33.

5. Delalande S, de Seze J, Fauchais AL, et al. Neurologic manifestations in primary Sjogren syndrome: a study of 82 patients. Medicine (Baltimore). 2004;83:280-91.

6. Alexander GE, Provost TT, Stevens MB, Alexander EL. Sjogren syndrome: central nervous system manifestations. Neurology. 1981;31:1391-6.

7. Basir A, Bougteba A, Kissani N. Symptomatic status epilepticus secondary to primary Sjogren's syndrome. Rev Neurol (Paris) 2010;166:108-10. 